$11-1-2008$

\title{
Analyzing Incomplete Categorical Data: Revisiting Maximum Likelihood Estimation (Mle) Procedure
}

Hoo Ling Ping

The University of Nottingham, lphoo_04@yahoo.com

M. Ataharul Islam

University of Dhaka, Bangladesh, mataharul@yahoo.com

Follow this and additional works at: http://digitalcommons.wayne.edu/jmasm

Part of the Applied Statistics Commons, Social and Behavioral Sciences Commons, and the Statistical Theory Commons

\section{Recommended Citation}

Ping, Hoo Ling and Islam, M. Ataharul (2008) "Analyzing Incomplete Categorical Data: Revisiting Maximum Likelihood Estimation (Mle) Procedure," Journal of Modern Applied Statistical Methods: Vol. 7 : Iss. 2 , Article 14.

DOI: $10.22237 /$ jmasm/1225512780

Available at: http://digitalcommons.wayne.edu/jmasm/vol7/iss2/14 


\section{Analyzing Incomplete Categorical Data: Revisiting Maximum Likelihood Estimation (Mle) Procedure}

\author{
Hoo Ling Ping \\ The University of Nottingham, \\ Malaysia
}

\author{
M. Ataharul Islam \\ University of Dhaka, \\ Bangladesh
}

Incomplete data poses formidable difficulties in the application of statistical techniques and requires special procedures to handle. The most common ways to solve this problem are by ignoring, truncating, censoring or collapsing those data, but these may lead to inappropriate conclusions because those data might contain important information. Most of the research for estimating cell probabilities involving incomplete categorical data is based on the EM algorithm. A likelihood approach is employed for estimating cell probabilities for missing values and makes comparisons between maximum likelihood estimation (MLE) and the EM algorithm. The MLE can provide almost the same estimates as that of the EM algorithm without any loss of properties. Results are compared for different distributional assumptions. Using clinical trial results from a group of 59 epileptics, results from the application of MLE and EM algorithm are compared and the advantages of MLE are highlighted.

Key words: Incomplete categorical data, maximum likelihood estimation (MLA), EM algorithm, multinomial distribution, binomial distribution, Poisson distribution, Newton-Raphson method.

\section{Introduction}

Incomplete data is referred to as data in which entries are missing, were a prior zero or are undetermined (Fienberg, 1980). Incomplete data is one of the main obstacles to researchers; this is especially true in the case of incomplete categorical data. The most common ways to solve this problem are by ignoring, truncating, censoring or collapsing those data; however, such procedures may lead to confusion and/or inappropriate conclusions because those data might contain important information.

Little \& Rubin (1987) defined the missing data mechanisms as ignorable missing data mechanism and non-ignorable missing data mechanism. The ignorable missing data mechanism involves process missing completely

Hoo Ling Ping is Assistant Professor in the Department of Applied Mathematics, Faculty of Engineering. Email: 1phoo_04@yahoo.com. M. Ataharul Islam is Professor in the Department of Statistics, Biostatistics and Informatics. Email: mataharul@yahoo.com. at random (MCAR) and missing at random (MAR). When the missingness is independent of both unobserved and observed data, the nonresponse process is named as MCAR. However, if the missingness is independent of the unobserved measurement conditionally on the observed data, the non-response process is called MAR. Non-ignorable missing data mechanisms involve informative process. When the process is neither MCAR nor MAR, then the process is termed informative. This article considers the missing data mechanism as a non-ignorable missing data mechanism.

The problem of estimation for incomplete contingency tables under the quasiindependence model was examined by Fienberg (1970), who used the maximum likelihood estimation (MLE) procedure. Similarly, MLE for the Poisson and Multinomial sampling distributions for the incomplete contingency tables in the presence of missing row and missing column data were considered by Chen \& Fienberg (1974). Chen \& Fienberg (1976) extended their works which focused on crossclassifications containing some totally mixed up cell frequencies with multinomial sampling. In the following year, Dempster, Laird \& Rubin 
presented MLE of incomplete data and named the algorithm EM since each iteration of the algorithm involved expectation (E) and maximization (M) steps. This method has been used extensively by other researchers especially for incomplete categorical data. Among others, Fuchs (1982), Nordheim (1984), Fay (1986), Baker \& Laird (1988), and Philips (1993) have used the EM algorithm for analyzing incomplete categorical data. The EM algorithm was used to improve the convergence of the EM by incorporating the Newton-Rapson approach by Baker (1994) and Galecki \& Molenberghs (2001). The EM algorithm is well developed (Lauritzen, 1995) to exploit the computational scheme of Lauritzen \& Spiegelhalter (1988) to perform the E-step of EM algorithm to find MLE in hierarchical log-linear models and recursive models for contingency tables with missing data. Molenberghs \& Goetchebeur (1997) presented a simple expression of the observed data log-likelihood for the EM algorithm. Tang, et. al. (2007) also found that the EM algorithm is the most widely used approach for finding the maximum likelihood estimate for incomplete data situations, but it lacks the direct provision of a measure of precision for the estimators and has a slow rate of convergence.

Because the EM algorithm was introduced, the MLE procedure was largely ignored by researchers until 1985. Stasny (1985) used MLE to process the model based on data from a Current Population Survey, and also used a Labor Force Survey to estimate gross flow data. Most recently, Lyles \& Allen (2003) proposed MLE with multinomial likelihood, properly accounting for missing data and assuming that the probability of missing exposure depends on true exposure.

In this article, not only is the missing row or missing column data redistributed, but also both row and column missing data for multinomial sampling by extending the works of Chen \& Fienberg (1974). Both row and column missing data are also investigated for the EM algorithm which has not been studied before. The MLE method for Poisson and Binomial sampling distributions was also examined as an extension of the works of Chen \& Fienberg (1974). The binomial distribution can be considered a special case of the Multinomial distribution. The same sampling patterns for the EM algorithm are considered here. The NewtonRaphson method was adopted in the MLE procedure to make convergence faster. Results of the MLE and EM algorithm are compared and the advantages of MLE are highlighted.

This article is organized as follows: data taken from Diggle, Liang \& Zeger (1994) is described, followed by the formulation of the MLE and EM algorithms. Finally, results are discussed, testing independence is presented and conclusions are put forth.

\section{Methodology}

The data considered herein was referred from Diggle, Liang \& Zeger (1994) based on a clinical trial of 59 epileptics. For each patient, the number of epileptic seizures was recorded during an eight week baseline period. Patients were then randomized to either a treatment group with anti-epileptic drug progabide $(0)$ or to a placebo group (1) and the number of seizures was recorded in four consecutive twoweek intervals. Table 1 shows the $2 \times 2$ artificial incomplete contingency table; rows refer to the treatment and columns refer to the results of the last treatment for the patient. The result of treatment is recorded as $\mathrm{Y}$.

Maximum likelihood estimation (MLE), Poisson and multinomial distribution

Chen \& Fienberg (1974) considered the MLE for incomplete contingency tables when missing row and column data existed. Their works are extended by considering incomplete contingency tables where either row or column, or both row and column are missing.

Let the fully cross-classified count for the $(i, j)^{\text {th }}$ cell of an $\mathrm{r}$ c contingency table be $x_{i j}$, $R_{i}(i=1,2, \ldots, r)$ is the count of the partially classified individuals corresponding to the $i^{\text {th }}$ row, $C_{j}(j=1,2, \ldots, c)$ is the count of the partially classified individuals corresponding to the $\mathrm{j}^{\text {th }}$ column, and $\mathrm{D}$ is the count of missing in both row and column. (See Figure 1.) Therefore the total sample size is:

$\mathrm{N}=\sum_{i j} x_{i j}+\sum_{i} R_{i}+\sum_{j} C_{j}+D=\mathrm{x}_{++}+\mathrm{R}_{+}+\mathrm{C}_{+}+\mathrm{D}$. 
Table1: Incomplete data

a) No missing on treatment and $\mathrm{Y}$

\begin{tabular}{|c|c|c|c|c|}
\cline { 3 - 5 } \multicolumn{2}{c|}{} & \multicolumn{2}{|c|}{$Y$} & \multirow{2}{*}{ Total } \\
\cline { 3 - 5 } \multicolumn{2}{c|}{} & $\leq 5$ & $>5$ & \\
\hline \multirow{2}{*}{ Treatment } & 0 & 13 & 7 & 20 \\
\cline { 2 - 5 } & 1 & 12 & 7 & 19 \\
\hline Total & 25 & 14 & 39 \\
\hline
\end{tabular}

b) Missing $Y$, treatment, $Y$ and treatment

\begin{tabular}{|c|c|c|}
\multicolumn{2}{c|}{} & Missing Y \\
\hline \multirow{2}{*}{ Treatment } & 0 & 3 \\
\cline { 2 - 3 } & 1 & 7 \\
\hline Total & 10 \\
\hline
\end{tabular}

\begin{tabular}{|c|cc|c|}
\cline { 2 - 3 } \multicolumn{1}{c|}{} & \multicolumn{2}{|c|}{ Y } & Total \\
\hline Missing & Yes & No & \\
Treatment & 2 & 2 & 4 \\
\hline
\end{tabular}

\begin{tabular}{|c|c|c|}
\hline & $\begin{array}{c}\text { Missing } \\
\text { Y }\end{array}$ & Total \\
\hline $\begin{array}{c}\text { Missing } \\
\text { Treatment }\end{array}$ & 6 & \\
\hline Total & & 6 \\
\hline
\end{tabular}

Figure 1: Illustration for complete observed and incomplete data

a) Complete observed data

\begin{tabular}{|c|c|c|c|c|c|}
\hline & \multicolumn{4}{|c|}{ Row } & Total \\
\hline \multirow{4}{*}{ Column } & $\mathrm{x}_{11}$ & $\mathrm{x}_{12}$ & $\ldots$ & $\mathrm{x}_{1 \mathrm{c}}$ & $\mathrm{x}_{1+}$ \\
\hline & $\mathrm{x}_{21}$ & $\mathrm{x}_{22}$ & $\ldots$ & $\mathrm{X}_{2 \mathrm{c}}$ & $2+$ \\
\hline & $\vdots$ & $\vdots$ & $\ddots$ & $\vdots$ & $\vdots$ \\
\hline & $\mathrm{X}_{\mathrm{r} 1}$ & $\mathrm{X}_{\mathrm{r} 2}$ & $\ldots$ & $\mathrm{X}_{\mathrm{rc}}$ & $\mathrm{X}_{\mathfrak{c}^{+}}$ \\
\hline Total & $\mathbf{x}_{+1}$ & $\mathrm{x}_{+2}$ & & $\mathrm{X}+\mathrm{c}$ & $\mathrm{x}$ \\
\hline
\end{tabular}

b) Incomplete units

\begin{tabular}{|c|c|}
\cline { 2 - 2 } \multicolumn{1}{c|}{} & Missing column \\
\hline Row & $\mathrm{R}_{1+}$ \\
& $\mathrm{R}_{2+}$ \\
& $\vdots$ \\
& $\mathrm{R}_{\mathrm{r}^{+}}$ \\
\hline Total & $\mathrm{R}$ \\
\hline
\end{tabular}

\begin{tabular}{|c|cc|c|c|c|}
\cline { 2 - 6 } \multicolumn{1}{c|}{} & \multicolumn{4}{c|}{ Column } & Total \\
\hline Missing row & $\mathrm{C}_{+1}$ & $\mathrm{C}_{+2}$ & $\ldots$ & $\mathrm{C}_{+\mathrm{c}}$ & $\mathrm{C}$ \\
\hline
\end{tabular}

\begin{tabular}{|c|c|c|}
\cline { 2 - 3 } \multicolumn{1}{c|}{} & Missing column & Total \\
\hline Missing row & $\mathrm{D}$ & \\
\hline Total & & $\mathrm{D}$ \\
\hline
\end{tabular}


When the original sampling scheme is Poisson with an expected value $m_{i j}$ for the $(i, j)^{\text {th }}$ cell, parameters associated with the cells (illustrated in Table 2) where $\lambda_{1(i)}, \lambda_{2(j)}$ and $\lambda$ are referred to the probabilities of losing its row, column, and both row and column identity, respectively. The cell probability of multinomial sampling for a completely classified (i, $\mathrm{j}^{\text {th }}$ cell is $\pi_{i j}$ and $\sum_{i} \sum_{j} \pi_{i j}=1$. By replacing $\pi_{i j}$ with $\mathrm{m}_{\mathrm{ij}}$, the likelihood function for Poisson is

$$
\begin{aligned}
& \exp \left(-\sum_{i} \sum_{j} m_{i j}\right) \prod_{i} \prod_{j}\left[\left(1-\lambda_{1(i)}-\lambda_{2(j)}-\lambda\right) m_{i j}\right]^{x_{i j}} \\
& \prod_{i}\left(\lambda_{1(i)} m_{i+}\right)^{R_{i}} \prod_{j}\left(\lambda_{2(j)} m_{+j}\right)^{C_{i}}\left(\lambda m_{++}\right)^{D}
\end{aligned}
$$

where

$$
\mathrm{m}_{\mathrm{i}+}=\sum_{i} m_{i j}, \mathrm{~m}_{+\mathrm{j}}=\sum_{j} m_{i j}, \text { and } \mathrm{m}_{++}=\sum_{i} \sum_{j} m_{i j}
$$

for all $\mathrm{i}$ and $\mathrm{j}$.

Equation (2) is a product of functions $f_{1}$ and $\mathrm{f}_{2}$ defined as follows

$$
\mathrm{f}_{1}=\frac{\left[\prod_{i} \prod_{j}\left(1-\lambda_{1(i)}-\lambda_{2(j)}-\lambda\right)^{x_{i j}}\right]}{\left[\prod_{i} \lambda_{1(i)}^{R_{i}}\right]\left[\prod_{j} \lambda_{2(j)}^{C_{j}}\right]\left[\lambda^{D}\right]}
$$

and

$$
\mathrm{f}_{2}=\begin{aligned}
& \exp \left(-\sum_{i} \sum_{j} m_{i j}\right)\left[\prod_{i} \prod_{j} m_{i j}^{x_{i j}}\right] . \\
& {\left[\prod_{i} m_{i+}^{R_{i}}\right]\left[\prod_{j} m_{+j}^{C_{j}}\right]\left[m_{++}^{D}\right]}
\end{aligned}
$$

when considering the unrestricted log linear model where

$$
\log \mathrm{m}_{\mathrm{ij}}=\mu+\alpha_{i}+\beta_{j}+\gamma_{i j} .
$$

Therefore,

$$
\begin{aligned}
\log \mathrm{f}_{2}= & -\sum_{i} \sum_{j} m_{i j}+\sum_{i} \sum_{j} x_{i j} \log m_{i j}+ \\
& \sum_{i} R_{i} \log m_{i+}+\sum_{j} C_{j} \log m_{+j}+D \log m_{++} \\
& -\sum_{i} \sum_{j} e^{\mu+\alpha_{i}+\beta_{j}+\gamma_{i j}}+\sum_{i} \sum_{j} x_{i j} \\
& \left(\mu+\alpha_{i}+\beta_{j}+\gamma_{i j}\right)+\sum_{i} R_{i}\left(\mu+\alpha_{i}+\beta_{+}+\gamma_{i+}\right)
\end{aligned}
$$

$$
\begin{aligned}
& +\sum_{j} C_{j}\left(\mu+\alpha_{+}+\beta_{j}+\gamma_{i+}\right) \\
& +D\left(\mu+\alpha_{+}+\beta_{+}+\gamma_{++}\right)
\end{aligned}
$$

Differentiating (6) with $\mu, \alpha_{i}, \beta_{j}$ and $\gamma_{i j}$,t, results in:

$$
\begin{aligned}
& \begin{aligned}
\frac{\partial \log f_{2}}{\partial \mu}= & -\sum_{i} \sum_{j} e^{\mu+\alpha_{i}+\beta_{j}+\gamma_{i j}} \\
& +\sum_{i} \sum_{j} x_{i j}+\sum_{i} R_{i}+\sum_{j} C_{j}+D
\end{aligned} \\
& =-\sum_{i} \sum_{j} m_{i j}+\sum_{i} \sum_{j} x_{i j}+\sum_{i} R_{i}+\sum_{j} C_{j}+D \\
& =-\mathrm{m}_{++}+\mathrm{x}_{++}+\mathrm{R}_{+}+\mathrm{C}_{+}+\mathrm{D} \\
& \frac{\partial \log f_{2}}{\partial \alpha_{i}}=-\mathrm{m}_{\mathrm{i}^{+}}+\mathrm{x}_{\mathrm{i}^{+}}+\mathrm{R}_{\mathrm{i}}+\sum_{j} C_{j}\left(\frac{m_{i j}}{m_{+j}}\right)+D\left(\frac{m_{i^{+}}}{m_{++}}\right) \\
& \frac{\partial \log f_{2}}{\partial \beta_{j}}=-\mathrm{m}_{+\mathrm{j}}+\mathrm{x}_{+\mathrm{j}}+\sum_{i} R_{i}\left(\frac{m_{i j}}{m_{i+}}\right)+\mathrm{C}_{\mathrm{j}}+D\left(\frac{m_{+j}}{m_{++}}\right) \\
& \frac{\partial \log f_{2}}{\partial \gamma_{i j}}=\mathrm{m}_{\mathrm{ij}}+\mathrm{x}_{\mathrm{ij}}+R_{i}\left(\frac{m_{i j}}{m_{i+}}\right)+C_{j}\left(\frac{m_{i j}}{m_{+j}}\right)+D\left(\frac{m_{i j}}{m_{++}}\right)
\end{aligned}
$$

When (7) is equal to 0 ,

$$
\hat{m}_{i j}=x_{i j}+R_{i}\left(\frac{\hat{m}_{i j}}{m_{i+}}\right)+C_{j}\left(\frac{\hat{m}_{i j}}{m_{+j}}\right)+D\left(\frac{\hat{m}_{i j}}{m_{++}}\right) .
$$

As from Chen \& Fienberg (1974), (8) is not able to be solved in closed from; initial estimates of the $\left\{\hat{m}_{i j}\right\}$ will be considered as

$$
m_{i j}^{(0)}=\left(\frac{x_{i j}}{x_{++}}\right) N .
$$

On the first iteration, from (8)

$$
\hat{m}_{i j}^{(1)}=x_{i j}+R_{i}\left(\frac{\hat{m}_{i j}^{(0)}}{m_{i+}^{(0)}}\right)+C_{j}\left(\frac{\hat{m}_{i j}^{(0)}}{m_{+j}^{(0)}}\right)+D\left(\frac{\hat{m}_{i j}^{(0)}}{m_{++}^{(0)}}\right),(
$$

therefore on $(\mathrm{k}+1)^{\text {th }}$ iteration,

$$
\begin{aligned}
& \hat{m}_{i j}^{(k+1)}=x_{i j}+R_{i}\left(\frac{\hat{m}_{i j}^{(k)}}{m_{i+}^{(k)}}\right)+C_{j}\left(\frac{\hat{m}_{i j}^{(k)}}{m_{+j}^{(k)}}\right)+D\left(\frac{\hat{m}_{i j}^{(k)}}{m_{++}^{(k)}}\right) \\
& \text { When } \mathrm{k} \rightarrow \infty,\left|\hat{m}_{i j}^{(k+1)}-\hat{m}_{i j}^{(k)}\right| \leq \varepsilon .
\end{aligned}
$$


Table 2: Underlying probabilities for a $2 \times 2$ table

Fully Classified Table

Row

Supplemental

Margin

\begin{tabular}{|l|l|}
\hline$\left(1-\lambda_{1(1)}-\lambda_{2(1)}-\lambda\right) \pi_{11}$ & $\left(1-\lambda_{1(1)}-\lambda_{2(2)}-\lambda\right) \pi_{12}$ \\
\hline$\left(1-\lambda_{1(2)}-\lambda_{2(1)}-\lambda\right) \pi_{21}$ & $\left(1-\lambda_{1(2)}-\lambda_{2(2)}-\lambda\right) \pi_{22}$ \\
\hline
\end{tabular}

\begin{tabular}{|l|}
\hline$\lambda_{1(1)} \pi_{1+}$ \\
\hline$\lambda_{1(2)} \pi_{2+}$ \\
\hline
\end{tabular}

Missing

row and column

Column

Supplemental

Margin

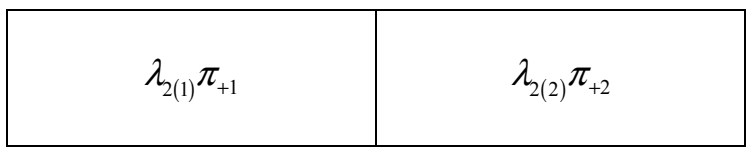

$\lambda \pi_{++}$

Since $\pi_{i j}=\frac{x_{i j}}{x_{++}}$for complete classified multinomial sampling, from (9) results,

$$
\hat{\pi}_{i j}^{(0)}=\frac{\hat{m}_{i j}^{(0)}}{N},
$$

and on the $(\mathrm{k}+1)^{\text {th }}$ iteration, $\hat{\pi}_{i j}^{(k+1)}=\frac{\hat{m}_{i j}^{(k+1)}}{N}$.

Poisson and binomial distributions

Now consider the complete contingency tables where there exist missing column. The fully cross-classified count for the $(i, j)$ cell of an $\mathrm{r} \times 2$ contingency table is $\mathrm{x}_{\mathrm{ij}}$, and $\mathrm{R}_{\mathrm{i}}(\mathrm{i}=1,2, \ldots$, $r)$ is the count of the partially classified individuals corresponding to the $i^{\text {th }}$ row. Therefore the total sample size is

$$
\begin{aligned}
\mathrm{N} & =\sum_{i j} x_{i j}+\sum_{i} R_{i} \\
& =\mathrm{x}_{++}+\mathrm{R}_{+}(12)
\end{aligned}
$$

When the original sampling scheme is Poisson with expected value $m_{i j}$ for the $(i, j)$ cell, then the parameters associated with the cells (illustrated in Table 3) where $\lambda_{1(i)}$, is referred to the probabilities of losing its row.

The cell probability of Binomial sampling for complete classified of ( $i, j$ ) cell is

$\pi_{i j}$ and $\sum_{i} \sum_{j} \pi_{i j}=1$. By replacing $\pi_{i j}$ with $\mathrm{m}_{\mathrm{ij}}$, the likelihood function for Poisson is

$$
\begin{aligned}
& \exp \left(-\sum_{i} \sum_{j} m_{i j}\right) \\
& \prod_{i} \prod_{j}\left[\left(1-\lambda_{\mathrm{I}(i)}\right) m_{i j}\right]^{x_{i j}} \prod_{i}\left(\lambda_{\mathrm{1}(i)} m_{i+}\right)^{R_{i}},
\end{aligned}
$$

where $\mathrm{m}_{\mathrm{i}+}=\sum_{i} m_{i j}$ for all $\mathrm{i}$ and $\mathrm{j}$.

Equation (13) is a product of a function

$$
\mathrm{f}_{1}=\left[\prod_{i} \prod_{j}\left(1-\lambda_{1(i)}\right)^{x_{i j}}\right]\left[\prod_{i} \lambda_{1(i)}^{R_{i}}\right]
$$

and

$$
\mathrm{f}_{2}=\exp \left(-\sum_{i} \sum_{j} m_{i j}\right)\left[\prod_{i} \prod_{j} m_{i j}^{x_{i j}}\right]\left[\prod_{i} m_{i+}^{R_{i}}\right] .
$$

When considering the unrestricted log linear model where

$$
\log \mathrm{m}_{\mathrm{ij}}=\mu+\alpha_{i}+\beta_{j}+\gamma_{i j},
$$

then, 
$\log \mathrm{f}_{2}=$

$-\sum_{i} \sum_{j} m_{i j}+\sum_{i} \sum_{j} x_{i j} \log m_{i j}$

$+\sum_{i} R_{i} \log m_{i+}$

$-\sum_{i} \sum_{j} e^{\mu+\alpha_{i}+\beta_{j}+\gamma_{i j}}+\sum_{i} \sum_{j} x_{i j}$

$=\left(\mu+\alpha_{i}+\beta_{j}+\gamma_{i j}\right)$

$+\sum_{i} R_{i}\left(\mu+\alpha_{i}+\beta_{+}+\gamma_{i+}\right)$

Differentiating (17) with $\mu, \alpha_{i}, \beta_{j}$ and $\gamma_{i j}$, results in

$$
\begin{gathered}
\frac{\partial \log f_{2}}{\partial \mu}=-\sum_{i} \sum_{j} e^{\mu+\alpha_{i}+\beta_{j}+\gamma_{i j}}+\sum_{i} \sum_{j} x_{i j}+\sum_{i} R_{i} \\
=-\sum_{i} \sum_{j} m_{i j}+\sum_{i} \sum_{j} x_{i j}+\sum_{i} R_{i} \\
=-\mathrm{m}_{++}+\mathrm{x}_{++}+\mathrm{R}_{+} \\
\frac{\partial \log f_{2}}{\partial \alpha_{i}}=-\mathrm{m}_{\mathrm{i}+}+\mathrm{x}_{\mathrm{i}+}+\mathrm{R}_{\mathrm{i}} \\
\frac{\partial \log f_{2}}{\partial \beta_{j}}=-\mathrm{m}_{+\mathrm{j}}+\mathrm{x}_{+\mathrm{j}}+\sum_{i} R_{i}\left(\frac{m_{i j}}{m_{+j}}\right) \\
\frac{\partial \log f_{2}}{\partial \gamma_{i j}}=-\mathrm{m}_{\mathrm{ij}}+\mathrm{x}_{\mathrm{ij}}+R_{i}\left(\frac{m_{i j}}{m_{i+}}\right)
\end{gathered}
$$

and, when (18) is equal to 0

$$
\hat{m}_{i j}=x_{i j}+R_{i}\left(\frac{\hat{m}_{i j}}{m_{i+}}\right) .
$$

Initial estimates of the $\left\{\hat{m}_{i j}\right\}$ were considered as

$$
m_{i j}^{(0)}=\left(\frac{x_{i j}}{x_{i+}}\right) m_{i+} .
$$

where $m_{i+}=x_{i+}+R_{i}$.

On the first iteration, from (19),

$$
\hat{m}_{i j}^{(1)}=x_{i j}+R_{i}\left(\frac{\hat{m}_{i j}^{(0)}}{m_{i+}}\right) .
$$

So, on the $(\mathrm{k}+1)^{\text {th }}$ iteration,

$$
\hat{m}_{i j}^{(k+1)}=x_{i j}+R_{i}\left(\frac{\hat{m}_{i j}^{(k)}}{m_{i+}}\right),
$$

when $\mathrm{k} \rightarrow \infty,\left|\hat{m}_{i j}^{(k+1)}-\hat{m}_{i j}^{(k)}\right| \leq \varepsilon$.

If an underlying Binomial sampling scheme is assumed, then

$$
p_{i j}=\frac{x_{i j}}{x_{i+}} .
$$

Therefore, from (20),

$$
\hat{p}_{i j}^{(0)}=\hat{m}_{i j}^{(0)} / m_{i+}
$$

and

$$
\hat{p}^{(0)}=\sum_{i} \sum_{j} \hat{m}_{i j}^{(0)} / N .
$$

On the $(\mathrm{k}+1)^{\text {th }}$ iteration,

$$
\hat{p}_{i j}^{(k+1)}=\hat{m}_{i j}^{(k+1)} / m_{i+}
$$

and

$$
\hat{p}^{(k+1)}=\sum_{i} \sum_{j} \hat{m}_{i j}^{(k+1)} / N .
$$

Table 3: Underlying probabilities for a $2 \times 2$ table

Fully Classified Table

\begin{tabular}{|l|l|}
\hline$\left(1-\lambda_{1(1)}\right) \pi_{11}$ & $\left(1-\lambda_{1(1)}\right) \pi_{12}$ \\
\hline$\left(1-\lambda_{1(2)}\right) \pi_{21}$ & $\left(1-\lambda_{1(2)}\right) \pi_{22}$ \\
\hline
\end{tabular}

Row

Supplemental

Margin

\begin{tabular}{|l|}
\hline$\lambda_{1(1)} \pi_{1+}$ \\
\hline$\lambda_{1(2)} \pi_{2+}$ \\
\hline
\end{tabular}


Formulation of Newton-Raphson

From Le (1992), the iterative solution for a parameter estimation on $(\mathrm{k}+1)^{\text {th }}$ iteration will be considered as

$$
\hat{\theta}^{(k+1)}=\hat{\boldsymbol{\theta}}^{(k)}+\Delta \hat{\boldsymbol{\theta}},
$$

where $\theta$ is the parameter and

$$
\Delta \hat{\theta}=-\left(\frac{d \ln L}{d \theta}\right) /\left(\frac{d^{2} \ln L}{d \theta^{2}}\right) .
$$

Differentiating (7) with $\gamma_{i j}$ and equal to 0 , then results in

$$
\begin{aligned}
m_{i j}= & R_{i+}\left[\frac{m_{i j}}{m_{i+}}-\left(\frac{m_{i j}}{m_{i+}}\right)^{2}\right] \\
& +C_{+j}\left[\frac{m_{i j}}{m_{+j}}-\left(\frac{m_{i j}}{m_{+j}}\right)^{2}\right]+D\left[\frac{m_{i j}}{m_{++}}-\left(\frac{m_{i j}}{m_{++}}\right)^{2}\right]
\end{aligned}
$$

To avoid the confusion of $m_{i j}$ for (7) and (24), let $m 1_{i j}$ and $m 2_{i j}$ for (7) and (24), respectively.

For application of the Newton-Raphson method in the two-way incomplete contingency table, consider

$$
\begin{aligned}
\hat{m} 1_{i j}^{(1)}=x_{i j}+R_{i+} & \left(\frac{\hat{m}_{i j}^{(0)}}{m_{i+}^{(0)}}\right)+C_{+j}\left(\frac{\hat{m}_{i j}^{(0)}}{m_{+j}^{(0)}}\right)+D\left(\frac{\hat{m}_{i j}^{(0)}}{m_{++}^{(0)}}\right) \\
m 2_{i j}^{(1)} & =R_{i+}\left[\frac{m 1_{i j}^{(0)}}{m 1_{i+}^{(0)}}-\left(\frac{m 1_{i j}^{(0)}}{m 1_{i+}^{(0)}}\right)^{2}\right] \\
+ & C_{+j}\left[\frac{m 1_{i j}^{(0)}}{m 1_{+j}^{(0)}}-\left(\frac{m 1_{i j}^{(0)}}{m 1_{+j}^{(0)}}\right)^{2}\right] . \\
+ & {\left[\frac{m 1_{i j}^{(0)}}{m 1_{++}^{(0)}}-\left(\frac{m 1_{i j}^{(0)}}{m 1_{++}^{(0)}}\right)^{2}\right] }
\end{aligned}
$$

where $\hat{m}_{i j}^{(0)}$ is the same with (9) and

$$
\hat{m}_{i j}^{(1)}=m 1_{i j}^{(0)}-\frac{m 1_{i j}^{(0)}}{m 2_{i j}^{(0)}} .
$$

On the $(\mathrm{k}+1)^{\text {th }}$ iteration,

$$
\begin{aligned}
\hat{m} 1_{i j}^{(k)} & =x_{i j}+R_{i+}\left(\frac{\hat{m}_{i j}^{(k-1)}}{m_{i+}^{(k-1)}}\right) \\
& +C_{+j}\left(\frac{\hat{m}_{i j}^{(k-1)}}{m_{+j}^{(k-1)}}\right)+D\left(\frac{\hat{m}_{i j}^{(k-1)}}{m_{++}^{(k-1)}}\right) .
\end{aligned}
$$

$$
\begin{aligned}
& m 2_{i j}^{(k)}= R_{i+}\left[\frac{m 1_{i j}^{(k-1)}}{m 1_{i+}^{(k-1)}}-\left(\frac{m 1_{i j}^{(k-1)}}{m 1_{i+}^{(k-1)}}\right)^{2}\right] \\
&+C_{+j}\left[\frac{m 1_{i j}^{(k-1)}}{m 1_{+j}^{(k-1)}}-\left(\frac{m 1_{i j}^{(k-1)}}{m 1_{+j}^{(k-1)}}\right)^{2}\right] \\
&+ {\left[\frac{m 1_{i j}^{(k-1)}}{m 1_{++}^{(k-1)}}-\left(\frac{m 1_{i j}^{(k-1)}}{m 1_{++}^{(k-1)}}\right)^{2}\right] } \\
& \hat{m}_{i j}^{(k+1)}=m 1_{i j}^{(k)}-\frac{m 1_{i j}^{(k)}}{m 2_{i j}^{(k)}} .
\end{aligned}
$$

For an accelerated convergence, these equations were employed to obtain the maximum likelihood estimators.

The EM algorithm: Formulation of the EM algorithm for contingency table

The EM approach for incomplete categorical data on the basis of Multinomial, Binomial and Poisson assumptions is now investigated.

Multinomial Distributions

For Multinomial distributions, the complete data log likelihood is

$$
\begin{gathered}
\log \mathrm{L}_{\mathrm{c}}\left(\pi_{\mathrm{i}}\right)=\sum_{i=1}^{n-1}\left(\mathrm{x}_{\mathrm{i}}+\mathrm{Z}_{\mathrm{i}}\right) \log \pi_{\mathrm{i}}+\left(\mathrm{x}_{\mathrm{n}}+\mathrm{z}_{\mathrm{n}}\right) \\
\log \left(1-\pi_{1}-\pi_{2}-\ldots-\pi_{n_{-1}}\right),
\end{gathered}
$$

where unobservable or missing data are referred to as $z_{i}=\left(z_{1}, z_{2}, \ldots, z_{n}\right)^{T}$ and $z_{i}=r_{i}+c_{i}+d_{i}$ with $r_{i}$ being missing column data, $c_{i}$ missing row data, and $\mathrm{d}_{\mathrm{i}}$ both row and column missing data on cell $\mathrm{i}^{\text {th }}$. Differentiating (31) with respect to $\pi_{\mathrm{i}}$, results in

$$
\hat{\pi}_{i}=\frac{x_{i}+z_{i}}{x_{n}+z_{n}} \pi_{n}
$$


Since $\sum_{i=1}^{n} \pi_{i}=1$, therefore from (32),

$$
\hat{\pi}_{i}=\frac{x_{i}+z_{i}}{N}
$$

where $\sum_{i=1}^{n}\left(x_{i}+z_{i}\right)=\mathrm{N}$.

The E- and M-values on the first iteration for cell $(i, j)$ were considered as follows.

E-step:

$$
m_{i j}^{(1)}=\mathrm{x}_{\mathrm{ij}}+\mathrm{R}_{\mathrm{i}+}\left(\frac{\pi_{i j}}{\pi_{i+}}\right)+\mathrm{C}_{+\mathrm{j}}\left(\frac{\pi_{i j}}{\pi_{+j}}\right)+\mathrm{D} \pi_{\mathrm{ij}}
$$

where $m_{i j}^{(1)}$ is the expected of cell $(i, j)$ on the first iteration.

M-step:

$$
\hat{\pi}_{i j}^{(1)}=m_{i j}^{(1)} / N,
$$

where $\pi_{i j}^{(1)}$ is the probability for cell $(\mathrm{i}, \mathrm{j})$.

On the $(\mathrm{k}+1)^{\text {th }}$ iteration, the E- and M-steps were defined as follows:

E-step:

$$
m_{i j}^{(k+1)}=\mathrm{x}_{\mathrm{ij}}+\mathrm{R}_{\mathrm{i}+}\left(\frac{\hat{\pi}_{i j}^{(k)}}{\hat{\pi}_{i+}^{(k)}}\right)+\mathrm{C}_{+\mathrm{j}}\left(\frac{\hat{\pi}_{i j}^{(k)}}{\hat{\pi}_{+i}^{(k)}}\right)+\mathrm{D} \pi_{\mathrm{ij}}
$$

M-step:

$$
\hat{\pi}_{i j}^{(k+1)}=m_{i j}^{(k+1)} / N
$$

The E- and M-steps were alternated and repeated until

$$
\begin{aligned}
\left|\hat{\pi}_{i j}^{(k+1)}-\hat{\pi}_{i j}^{(k)}\right| & =\left|\frac{m_{i j}{ }^{(k+1)}}{N}-\frac{m_{i j}{ }^{(k)}}{N}\right| \\
& =\left|\frac{m_{i j}{ }^{(k+1)}-m_{i j}{ }^{(k)}}{N}\right| \leq \varepsilon .
\end{aligned}
$$

Therefore, when $\mathrm{k} \rightarrow \infty, \lim _{k \rightarrow \infty}\left|\hat{\pi}_{i j}^{(k+1)}-\hat{\pi}_{i j}^{(k)}\right|=0$, and $\hat{\pi}_{i j}^{(k+1)}=\hat{\pi}_{i j}^{(k)}=\pi^{*}$.

Binomial distribution

For the binomial distribution, the complete-data log likelihood is

$\log \mathrm{L}_{\mathrm{c}}\left(p_{i 1}\right)=\left(\mathrm{x}_{\mathrm{i} 1}+\mathrm{Z}_{\mathrm{i} 1}\right) \log p_{i 1}+\left(\mathrm{x}_{\mathrm{i} 2}+\mathrm{z}_{\mathrm{i} 2}\right) \log \left(1-p_{i 1}\right)$, for $\mathrm{i}=1, \ldots, \mathrm{n}$, and $\mathrm{z}_{\mathrm{i}}$ is referred to as unobservable or missing data on the $i^{\text {th }}$ row where $\mathrm{z}_{\mathrm{i} 1}+\mathrm{z}_{\mathrm{i} 2}=\mathrm{z}_{\mathrm{i}}$. Differentiating with respect to $p_{i 1}$ results in

$$
\hat{p}_{i 1}=\frac{x_{i 1}+z_{i 1}}{x_{i 1}+x_{i 2}+z_{i}} .
$$

From (34), if all rows are summed, the following is obtained

$$
\sum_{i=1}^{I} \hat{p}_{i 1}=\frac{\sum_{i=1}^{I} r_{i 1}+z_{i 1}}{\sum_{i=1}^{I} r_{i 1}+r_{i 2}+z_{i}} .
$$

Since $\sum_{i=1}^{I} r_{i 1}+r_{i 2}+z_{i}=N$, total sample,

$$
\hat{p}_{+j}=\frac{\sum_{i=1}^{n} x_{i j}^{(1)}}{N} .
$$

The E- and M-values on the first iteration for cell $(i, j)$ were considered as follows:

E-step:

$$
m_{i j}^{(1)}=\mathrm{x}_{\mathrm{ij}}+\mathrm{R}_{\mathrm{i}+\mathrm{p}_{\mathrm{ij}}}
$$

where $m_{i j}^{(1)}$ is the expected value of cell $(i, j)$ on the first iteration and $\mathrm{p}_{\mathrm{ij}}=\mathrm{x}_{\mathrm{ij}} / \mathrm{x}_{\mathrm{i}+}$.

M-step:

$$
\hat{p}_{i j}^{(1)}=m_{i j}^{(1)} /\left(m_{i 1}^{(1)}+m_{i 2}^{(1)}\right),
$$

and

$$
\hat{p}_{+j}^{(1)}=\frac{\sum_{i=1}^{n} m_{i j}^{(1)}}{N}
$$

On the $(\mathrm{k}+1)^{\text {th }}$ iteration, the E- and M-steps were defined as follows: 
E-step:

$$
m_{i j}^{(k+1)}=\mathrm{x}_{\mathrm{ij}}+\mathrm{R}_{\mathrm{i}+} p_{i j}^{(k)} .
$$

M-step:

$$
\hat{p}_{i j}^{(k+1)}=m_{i j}^{(k+1)} /\left(m_{i 1}^{(k+1)}+m_{i 2}^{(k+1)}\right),
$$

and

$$
\hat{p}_{+j}^{(k+1)}=\frac{\sum_{i=1}^{n} m_{i j}^{(k+1)}}{N} .
$$

The E- and M-steps were alternated and repeated until

$$
\left|\hat{p}_{i j}^{(k+1)}-\hat{p}_{i j}^{(k)}\right|=\left|\frac{m_{i j}^{(k+1)}}{m_{i 1}^{(k+1)}+m_{i 2}^{(k+1)}}-\frac{m_{i j}^{(k)}}{m_{i 1}^{(k)}+m_{i 2}^{(k)}}\right| \leq \varepsilon,
$$

and

$$
\begin{aligned}
\left|\hat{p}_{+j}^{(k+1)}-\hat{p}_{+j}^{(k)}\right| & =\left|\frac{\sum_{i=1}^{n} m_{i j}^{(k+1)}}{N}-\frac{\sum_{i=1}^{n} m_{i j}^{(k)}}{N}\right| \\
& =\left|\frac{\sum_{i=1}^{n} m_{i j}^{(k+1)}-\sum_{i=1}^{n} m_{i j}^{(k)}}{N}\right| \leq \varepsilon .
\end{aligned}
$$

Therefore, when $\mathrm{k} \rightarrow \infty, \lim _{k \rightarrow \infty}\left|\hat{p}_{i j}^{(k+1)}-\hat{p}_{i j}^{(k)}\right|=0$ and $\lim _{k \rightarrow \infty}\left|\hat{p}_{+j}^{(k+1)}-\hat{p}_{+j}^{(k)}\right|=0$.

Poisson distribution

For the Poisson distribution, the complete-data log likelihood is

$\log \mathrm{L}_{\mathrm{c}}\left(\mathrm{y} ; \theta_{\mathrm{i}}\right)=$

$$
\sum_{i=1}^{n}\left[\left(x_{i}+z_{i}\right) \log \left(\theta_{i}\right)-\theta_{i}-\log \left(x_{i}+z_{i}\right) !\right]
$$

where $\mathrm{z}_{1}+\mathrm{z}_{2}+\ldots+\mathrm{z}_{\mathrm{n}}$ is referred to as unobservable or missing data. By differentiating (35) with respect to $\theta_{\mathrm{i}}$,

$$
\hat{\theta}_{i}=x_{i}+z_{i} .
$$

Referring to Figure 1, the E- and Mvalues on the first iteration for the cell $(i, j)$ was considered as:
E-step:

$$
z_{i j}^{(1)}=R_{i j}^{(1)}+C_{i j}^{(1)}+D_{i j}^{(1)},
$$

where

$$
\begin{gathered}
R_{i j}^{(1)}=\mathrm{R}_{\mathrm{i}+}\left(\frac{x_{i j}}{x_{i+}}\right), C_{i j}^{(1)}=\mathrm{C}_{+\mathrm{j}}\left(\frac{x_{i j}}{x_{+j}}\right), \text { and } \\
D_{i j}^{(1)}=\mathrm{D}\left(\frac{x_{i j}}{x}\right) .
\end{gathered}
$$

M-step:

$$
\hat{\theta}_{i j}^{(1)}=x_{i j}+z_{i j}^{(1)} .
$$

On the $(\mathrm{k}+1)^{\text {th }}$ iteration, the E- and M-steps were defined as follows:

E-step:

$$
z_{i j}^{(k+1)}=R_{i j}^{(k+1)}+C_{i j}^{(k+1)}+D_{i j}^{(k+1)},
$$

where

$$
\begin{gathered}
R_{i j}^{(k+1)}=\mathrm{R}_{\mathrm{i}+}\left(\frac{\theta_{i j}^{k}}{\theta_{i+}^{k}}\right), C_{i j}^{(k+1)}=\mathrm{C}_{+\mathrm{j}}\left(\frac{\theta_{i j}^{k}}{\theta_{+j}^{k}}\right), \text { and } \\
D_{i j}^{(k+1)}=\mathrm{D}\left(\frac{\theta_{i j}^{k}}{N}\right),
\end{gathered}
$$

and $\mathrm{N}$ is total sample.

M-step:

$$
\hat{\theta}_{i j}^{(1)}=x_{i j}+z_{i j}^{(1)} .
$$

The E- and M-steps were alternated and repeated until

$$
\begin{aligned}
\left|\hat{\theta}_{i j}^{(k+1)}-\hat{\theta}_{i j}^{(k)}\right| & =\left|\left(x_{i j}+z_{i j}^{(k+1)}\right)-\left(x_{i j}+z_{i j}^{(k)}\right)\right| \\
& =\left|z_{i j}^{(k+1)}-z_{i j}^{(k)}\right| \leq \varepsilon
\end{aligned}
$$

Therefore, when $\mathrm{k} \rightarrow \infty$

$$
\lim _{k \rightarrow \infty}\left|\hat{\theta}_{i j}^{(k+1)}-\hat{\theta}_{i j}^{(k)}\right|=0
$$

and it may be said that $\hat{\theta}_{i j}^{(k+1)}=\hat{\theta}_{i j}^{(k)}=\theta^{*}$.

\section{Results}

The results of MLE, adopting Newton-Raphson in MLE and the M-step of the EM algorithm for the Poisson distribution are presented in Tables 4, 5 and 6 respectively. 
Table 4 MLE for Poisson distribution

\begin{tabular}{|c|c|c|c|c|}
\hline \multirow{2}{*}{ Iteration } & \multicolumn{4}{|c|}{ Cells } \\
\cline { 2 - 5 } & $(1,1)$ & $(1,2)$ & $(2,1)$ & $(2,2)$ \\
\hline 1 & 17.99 & 10.13 & 19.23 & 11.66 \\
\hline 2 & 17.72 & 10.05 & 19.35 & 11.89 \\
\hline 3 & 17.67 & 10.03 & 19.35 & 11.96 \\
\hline 4 & 17.66 & 10.02 & 19.34 & 11.98 \\
\hline 5 & 17.67 & 10.02 & 19.33 & 11.99 \\
\hline 6 & 17.67 & 10.01 & 19.33 & 11.99 \\
\hline 7 & 17.67 & 10.01 & 19.33 & 11.99 \\
\hline
\end{tabular}

Table 5: Adopting Newton-Raphson in MLE for Poisson distribution

\begin{tabular}{|c|c|c|c|c|}
\hline \multirow{2}{*}{ Iteration } & \multicolumn{4}{|c|}{ Cells } \\
\cline { 2 - 5 } & $(1,1)$ & $(1,2)$ & $(2,1)$ & $(2,2)$ \\
\hline 1 & 17.92 & 9.7 & 19.62 & 11.76 \\
\hline 2 & 17.68 & 9.38 & 19.87 & 12.06 \\
\hline 3 & 17.66 & 9.34 & 19.78 & 12.22 \\
\hline 4 & 17.65 & 9.34 & 19.77 & 12.24 \\
\hline 5 & 17.65 & 9.34 & 19.77 & 12.24 \\
\hline
\end{tabular}

Table 6: M-step for Poisson distribution

\begin{tabular}{|c|c|c|c|c|}
\hline \multirow{2}{*}{ Iteration } & \multicolumn{4}{|c|}{ Cells } \\
\cline { 2 - 5 } & $(1,1)$ & $(1,2)$ & $(2,1)$ & $(2,2)$ \\
\hline 1 & 17.99 & 10.13 & 19.22 & 11.66 \\
\hline 2 & 17.72 & 10.04 & 19.35 & 11.9 \\
\hline 3 & 17.67 & 10.02 & 19.35 & 11.96 \\
\hline 4 & 17.67 & 10.02 & 19.34 & 11.98 \\
\hline 5 & 17.67 & 10.01 & 19.33 & 11.98 \\
\hline 6 & 17.67 & 10.01 & 19.33 & 11.99 \\
\hline 7 & 17.67 & 10.01 & 19.33 & 11.99 \\
\hline
\end{tabular}

The results of MLE, adopting NewtonRaphson in MLE and the M-step of the EM algorithm for the Multinomial distribution are presented in Tables 7, 8 and 9 respectively. The results of MLE and the M-step for the Binomial distribution are presented in Tables 10, 11 and 12 respectively.

Based upon results, both the MLE and the EM algorithms converge on the $7^{\text {th }}$ iteration (see Tables 4 and 6), and both methods give the same results. However, by adopting the NewtonRaphson in the MLE, the results on the $5^{\text {th }}$ iteration were obtained (see Table 5). Although it seems that the EM algorithm was converging the same as the MLE, the EM algorithm involves two calculation steps on each iteration. 
In other words, the EM takes longer to compute the results compared with the MLE. After adopting the Newton-Raphson in the MLE, it was able to give faster convergence without as much deviance in the results as the EM algorithm. Tables 7 and 8 were obtained by considering the last iteration of Tables 4 and 5 respectively. The results were also the same for the Multinomial distribution for the MLE and the EM algorithm. By comparing the results of Table 8 with the last iteration of Table 9, it is observed that the results are not much different. However Table 11 was obtained by considering the last iteration of Table 10. Results shown in Tables 11 and 12 were the same as those obtained for the Binomial distribution.

Testing independence

For two-way contingency tables, the null hypothesis of statistical independence is $\mathrm{H}_{0}$ $: \pi_{\mathrm{ij}}=\pi_{\mathrm{i}^{+}} \pi_{+_{\mathrm{j}}}$ for all $\mathrm{i}$ and $\mathrm{j}$. The likelihood-ratio statistic, $\mathrm{G}^{2}$ is asymptotically equivalent to $\chi^{2}$ when $\mathrm{n} \rightarrow \infty$ with d.f. $=(\mathrm{r}-1)(\mathrm{c}-1)$ where $\mathrm{r}$ is the number of rows and $c$ is the number of columns in the contingency table.

According to Schafer (1997), $\mathrm{G}^{2}=$ $2\left[\ell\left(\hat{\pi} \mid Y_{o b s}\right)-\ell\left(\tilde{\pi} \mid Y_{o b s}\right)\right]$, where $\ell\left(\hat{\pi} \mid Y_{o b s}\right)$ is the unrestricted ML estimate $(\hat{\pi})$ and $\ell\left(\tilde{\pi} \mid Y_{o b s}\right)$ is the restricted ML estimate $(\tilde{\pi})$. Thus,

Table 7: MLE for the Multinomial distribution

\begin{tabular}{|c|c|c|c|}
\hline \multicolumn{4}{|c|}{ Cells } \\
\hline$(1,1)$ & $(1,2)$ & $(2,1)$ & $(2,2)$ \\
\hline 0.2992 & 0.1697 & 0.3276 & 0.2032 \\
\hline
\end{tabular}

Table 8: Adopting Newton-Raphson in MLE for the Multinomial distribution

\begin{tabular}{|c|c|c|c|}
\hline \multicolumn{4}{|c|}{ Cells } \\
\hline$(1,1)$ & $(1,2)$ & $(2,1)$ & $(2,2)$ \\
\hline 0.2992 & 0.1583 & 0.3351 & 0.2075 \\
\hline
\end{tabular}

$\ell\left(\pi \mid Y_{o b s}\right)$ is considered as:

$$
\begin{gathered}
\ell\left(\pi \mid Y_{o b s}\right)=\ell_{A}\left(\pi \mid Y_{o b s}\right)+\ell_{B}\left(\pi \mid Y_{o b s}\right) \\
+\ell_{C}\left(\pi \mid Y_{o b s}\right)+\ell_{D}\left(\pi \mid Y_{o b s}\right) .
\end{gathered}
$$

For the Multinomial and Poisson distributions with the MLE and EM algorithm,

$$
\mathrm{G}^{2}=2\left[\ell\left(\hat{\pi} \mid Y_{o b s}\right)-\ell\left(\tilde{\pi} \mid Y_{o b s}\right)\right] .
$$

For the Binomial distribution, $\mathrm{H}_{0}: \mathrm{p}=\mathrm{p}_{\mathrm{i} 1}$, therefore

$$
\mathrm{G}^{2}=2\left[\ell\left(\hat{p} \mid Y_{o b s}\right)-\ell\left(\tilde{p} \mid Y_{o b s}\right)\right],
$$

where $\ell\left(\hat{p} \mid Y_{o b s}\right)$ is the unrestricted ML estimate of $\hat{p}$ and $\ell\left(\tilde{p} \mid Y_{o b s}\right)$ is the restricted ML estimate of $\tilde{p}$. For both the MLE and the EM algorithms $\ell\left(p \mid Y_{o b s}\right)$ is considered as:

$$
\ell\left(p \mid Y_{o b s}\right)=\ell_{A}\left(p \mid Y_{o b s}\right)+\ell_{B}\left(p \mid Y_{o b s}\right) .
$$

Therefore, adopting the NewtonRaphson in the MLE and EM algorithms for Multinomial and Poisson distributions, $\mathrm{G}^{2}=0.02$. However, for the Binomial distribution, $\mathrm{G}^{2}=0.01$. From these results, it may be concluded that treatment type is independent of the results of treatment for the Multinomial and Poisson distributions, and the number of seizure pain which is less than five is the same for treatment 0 and 1 for the Binomial distribution.

Table 9: M-step for Multinomial distribution

\begin{tabular}{|c|c|c|c|c|}
\hline \multirow{2}{*}{ Iteration } & \multicolumn{4}{|c|}{ Cells } \\
\cline { 2 - 5 } & $(1,1)$ & $(1,2)$ & $(2,1)$ & $(2,2)$ \\
\hline 1 & 0.3049 & 0.1716 & 0.3259 & 0.1976 \\
\hline 2 & 0.3003 & 0.1702 & 0.3278 & 0.2017 \\
\hline 3 & 0.2995 & 0.1698 & 0.3278 & 0.2027 \\
\hline 4 & 0.2993 & 0.1698 & 0.3278 & 0.2031 \\
\hline 5 & 0.2992 & 0.1697 & 0.3276 & 0.2031 \\
\hline 6 & 0.2992 & 0.1697 & 0.3276 & 0.2032 \\
\hline 7 & 0.2992 & 0.1697 & 0.3276 & 0.2032 \\
\hline
\end{tabular}


Table 10: MLE for the Poisson distribution

\begin{tabular}{|c|c|c|c|c|}
\hline \multirow{2}{*}{ Iteration } & \multicolumn{4}{|c|}{ Cells } \\
\cline { 2 - 5 } & $(1,1)$ & $(1,2)$ & $(2,1)$ & $(2,2)$ \\
\hline 1 & 14.95 & 8.05 & 16.42 & 9.58 \\
\hline 2 & 14.95 & 8.05 & 16.42 & 9.58 \\
\hline
\end{tabular}

Table 11: MLE for the Binomial distribution

\begin{tabular}{|c|c|c|c|c|c|}
\hline \multicolumn{6}{|c|}{ Cells } \\
\hline$(1,1)$ & $(2,1)$ & $(+, 1)$ & $(1,2)$ & $(2,2)$ & $(+, 2)$ \\
\hline 0.65 & 0.6315 & 0.6402 & 0.35 & 0.3685 & 0.3598 \\
\hline
\end{tabular}

Table 12: M-step for the Binomial distribution

\begin{tabular}{|c|c|c|c|c|c|c|}
\hline \multirow{2}{*}{ Iteration } & \multicolumn{6}{|c|}{ Cells } \\
\cline { 2 - 7 } & $(1,1)$ & $(2,1)$ & $(+, 1)$ & $(1,2)$ & $(2,2)$ & $(+, 2)$ \\
\hline 1 & 0.65 & 0.6315 & 0.6402 & 0.35 & 0.3685 & 0.3598 \\
\hline 2 & 0.65 & 0.6315 & 0.6402 & 0.35 & 0.3685 & 0.3598 \\
\hline
\end{tabular}

\section{Conclusion}

The EM algorithm is more complicated than the MLE, because the EM algorithm involves the E(expectation) and M-(maximization) steps. This makes the calculations more complicated and also increases the amount of time required to calculate results as compared with the MLE, which is more straightforward for estimating cell probabilities in cases of incomplete categorical data. For example when consider a contingency table with a Poisson sampling scheme, for MLE, the expected value is obtained as in (11) by considering the previous iteration of the expected value. However, for the EM algorithm, before calculating the expected value in the $\mathrm{M}$ step, the E-step - which involves the estimation of initial cell probability first - must first be considered. For the Binomial sampling scheme, the convergence for estimation of $p_{i 1}$ and $p$ can be obtained when first considering Poisson sampling employing the MLE procedure. Again, if the EM algorithm is considered, the E-step is required first in order to obtain an initial estimate for $\mathrm{p}_{\mathrm{ij}}$. Similar explanations may be given for Multinomial sampling cases where, if MLE is considered, the Poisson sampling must be addressed before using the last iteration to obtain $\hat{\pi}_{i j}$. The EM algorithm, however, requires step by step convergence starting from the initial value for $\hat{\pi}_{i j}$ before convergence is achieved.

The MLE can better perform by adopting the Newton-Raphson method, because this method helps to accelerate the convergence. When the MLE is adopted with that of NewtonRaphson, as a convergence method, it is clear that the MLE and the EM algorithm are two different kinds of algorithms. The MLE algorithm provides a direct way to maximize the final expected value, while the EM algorithm involves expectation before the maximization; however, the EM algorithm demonstrates the distribution of missing values at each step until convergence on the basis of the marginal probabilities.

The MLE is much simpler than the EM algorithm when one is interested simply in final results. If interest lies in understanding the distribution of missing values in more detail, the EM algorithm is the better choice. 


\section{References}

Baker, S. G. (1994). Composite linear models for incomplete multinomial data. Statist. Med., 13, 609-622.

Baker, S. G., \& Laird, N. M. (1988). Regression analysis for categorical variables with outcome subject to nonignorable nonresponse. Journal of the American Statistical Association, 83, 62-69.

Bishop, Y. M. M., Fienberg, S. E., \& Holland, P. W. (1975). Discrete Multivariate Analysis:Theory and Practice. MIT Press: Cambridge, MA.

Chen, T., \& Fienberg, S. E. (1974). Two-dimensional contingency tables with both completely and partially cross-classified data. Biometrics, 30, 629-642.

Chen, T., \& Fienberg, S. E. (1976). The analysis of contingency tables with incompletely classified data. Biometrics, 32, 133-144.

Dempster, A. P., Laird, N. M., \& Rubin, D. B. (1977). Maximum likelihood from incomplete data via the EM algorithm (with discussion). J. Roy. Statist. Soc. B, 39, 1-38.

Diggle, P.J., Liang K. Y., \& Zeger S. L. (1994) Analysis of Longitudinal Data. Oxford University Press: Oxford, England.

Fay, R. E. (1986). Causal models for patterns of nonresponse. Journal of the American Statistical Association, 81, 354-365.

Fienberg, S. E. (1970). Quasiindependence and maximum likelihood estimation in incomplete contingency tables. Journal of the American Statistical Association, 65, 1610-1616.

Fienberg, S. E. (1980). The analysis of cross-classified categorical data. MIT Press: Cambridge, MA.

Fuchs, C. (1982). Maximum likelihood estimation and model selection in contingency tables with missing data. Journal of the American Statistical Association, 77, 270-278.

Galecki, A. T., Have, T. R. T. \& Molenberghs, G. (2001). A simple and fast alternative to the EM algorithm for incomplete categorical data and latent class models. Comp. Stat. \& Data Analysis, 35, 265-281.
Lauritzen, S. L. (1995). The EM algorithm for graphical association models with missing data. Comp. Stat. \& Data Analysis, 19, 191-201.

Lauritzen, S. L., \& Spiegelhalter, D. J. (1988). Local computations with probabilities on graphical structures and their application to expert systems (with discussion). Journal of the Royal Statistical Society B, 50, 157-224.

Le, C. T. (1992). Fundamentals of Biostatistical Inference. Marcel Dekker, Inc.: New York, NY.

Little, R. J. A., \& Rubin, D. B. (1987). Statistical analysis with missing data. John Wiley: New York, NY.

Little, R. J. A., \& Rubin, D. B. (2002). Statistical analysis with missing data, $2^{\text {nd }} E d$. John Wiley: New Jersey.

Lyles, R. H., \& Allen, A. S. (2003). Missing data in the $2 \times 2$ table: patterns and likelihood-based analysis for cross-sectional studies with supplemental sampling Stats. Med., 22, 517-534.

McLachlan, G. J., \& Krishnan, T. (1997). The EM algorithm and extensions. John Wiley: New York, NY.

Molenberghs, G., \& Goetchebeur, E. (1997). Simple fitting algorithm for incomplete categorical data. Journal of the Royal Statistical Society B, 59, 401-414.

Nordheim, E. V. (1984). Inference from nonrandomly missing categorical data: an example from a genetic study on Turner's syndrome. Journal of the American Statistical Association, 79, 772-780.

Phillips, M. J. (1993). Contingency tables with missing data, The Statistician, 42, 918.

Schafer, J. L. (1997). Analysis of incomplete multivariate data. Chapman and Hall/CRC: New York, NY.

Stasny, E. A. (1985). Modeling nonignorable non-response in panel data. Proceedings of the Survey Research Methods Section, American Statistical Association, 349354.

Tang, M. L., Ng, K. W., Tian, G. L. \& Tan, M. (2007). On improved EM algorithm and confidence interval construction for incomplete rxc tables. Computational Statistics and Data Analysis, 51: 2919-2933. 